\title{
TIME DELAY SYSTEMS - MEROMORPHIC FUNCTIONS CONTROL APPROACH
}

\author{
Roman Prokop, Libor Pekař \\ Faculty of Applied Informatics, Tomas Bata University in Zlin \\ Nad Stráněmi4511, 76005 Zlin, Czech Republic \\ Tel.:+420576035257 \\ E-mail:Pekar@fai.utb.cz.
}

\begin{abstract}
The paper develops an engineering acceptable approach to anisochronic controllers. The method is based on algebraic tools in the ring of RQ-meromorphic functions and it was developed for a wide class of delayed systems. This contribution deals with so-called anisochronic systems which include delays also in dynamics. Both stable and unstable systems are assumed. The control synthesis consists in the solution of the Bèzout identity and the Youla-Kučera parameterization resulting in the Smith-like control structure. A final controller can be tuned by a suitable choice of a scalar real parameter. Among many others tuning methods, the equalization method is adopted. The approach is suitable also for high order dynamics approximation and autotuning procedures. First order stable and unstable simulation examples are presented.
\end{abstract}

Keywords: Algebraic approaches, Delay compensation, Diophantine equation, Parameterization, Smith predictor.

\section{INTRODUCTION}

A family of systems with delays also in internal feedback loops constitutes an attractive and interesting branch for theory as well as for industrial application. Delays are traditionally modeled in the sense of input-output relations. This approach is not suitable for state delays, i.e. on the left side of a differential equation (Zítek and Víteček, 1999; Zítek and Kučera, 2003). However, different use of delay relations as one of the primary elements of model structure can be considered - anisochronic system description comprehending delay terms also in the state variables can cover a wide class of time delay systems. The models have transcendental structure, i.e. with infinite spectrum. There are several ways of identifying a plant with an anisochronic model, e.g. via relay test (Vyhlídal and Zítek, 2001) or successive integrations (Zítek and Vyhlídal, 2003). The algebraic description of anisochronic systems requires a simultaneous use of both differential and delay operators. The Laplace-transform description of this class of systems results in the transfer functions that are ratios of the so-called quasipolynomials. Once a ring of quasipolynomials is established, a set of retarded quasipolynomial meromorphic functions $\left(R_{M S}\right)$ can be introduced. Any transfer function is then described as a ratio of two elements from $R_{M S}$ and this representation is suitable for algebraic controller design.

Algebraic approaches play a significant role in modern control theory. Recently, some attempts have been made to implement algebraic controller design 
algorithms over the $R_{M S}$ ring (Zítek and Kučera, 2003; Zítek et al., 2005; Prokop et al , 2006). Two ideas predominate: functional extension of the internal model control principle using affine parameterization and solution based on Diophantine equations and the Youla-Kučera parameterization. With the only exception (Zítek et al., 2005), the approaches cover only stable controlled systems. This contribution develops the second mentioned approach which covers also controller design for unstable plants. The methodology brings a scalar parameter $m_{0}$ which can be used for controller tuning. The question how to choose the "right" value of this parameter has not been solved, one attempt is suggested in (Zítek and Vyhlídal, 2002) where dominant closed loop poles shifting method was utilized. This contribution brings another choice employing an analogy with the equalization method (Klán, 2005). The paper also demonstrates some simulation examples to verify the proposed algebraic controller design approach for various plant models.

\section{DESCRIPTION OF DELAYED SYSTEMS USING MEROMORPHIC FUNCTIONS}

A transfer function is obviously assumed as a ratio of two polynomials in the Laplace transform. A time delay in a system with the input-output delay where the dynamics is expressed by accumulations only is then expressed by an exponential

$$
\begin{aligned}
& G(s)=\frac{Y(s)}{U(s)}=\frac{b_{m} s^{m}+\ldots+b_{1} s+b_{0}}{s^{n}+\ldots+a_{1} s+a_{0}} e^{-\tau s}=\frac{b(s)}{a(s)} e^{-\tau s} ; \\
& m<n, \tau>0
\end{aligned}
$$

The innovative approach can be found e.g. in (Kamen, 1978; Zítek and Víteček, 1999) utilizing delays also in dynamics, i.e. in a denominator of a transfer function, providing a tighter model for a wide class of plants. Due to the infinite spectrum of the models they can be used for tracing of higher order systems dynamics. For the modeling both inductive (e.g. Vyhlídal and Zítek, 2001; Zítek and Vyhlídal, 2003) and deductive (Zítek and Víteček, 1999) procedures can be utilized. Using Laplace, transfer functions result in a ration of quasipolynomials. Quasipolynomials contain also exponentials terms, in contrast to polynomials which consists of weighted sums of s-powers only. As an example, the first order anisochronic system can be depicted as

$$
\tilde{G}(s)=\frac{b_{0} e^{-\tau s}}{s+a_{0} e^{-\theta_{s}}}
$$

Modern algebraic approaches utilize various rings and tools connected with their properties. Assume a ring of stable and proper retarded quasipolynomial (RQ) meromorphic functions $\left(R_{M S}\right)$ rather than quasipolynomials. This representation allows convenient using of parameterization providing that the specific control conditions are fulfilled. Considering transfer function (2), the plant description in $R_{M S}$ is as follows

$$
\tilde{G}(s)=\frac{\frac{b_{0} e^{-\tau s}}{s+m_{0} e^{-\theta s}}}{\frac{s+a_{0} e^{-\theta s}}{s+m_{0} e^{-\theta s}}}
$$

where analogously for higher order plants a common denominator is stable polynomial or quasipolynomial of appropriate order. Using of quasipolynomial rather than the polynomials is necessary in case of unstable delayed plants (Pekař, 2005). Quasipolynomial stability can be easily tested by applying the Mikhailov criterion, which was proved to be applicable for anisochronic systems (2) in Zítek (1997).

The issue of properness in $R_{M S}$ ring is as the natural requirement as in the rational descriptions ensuring feasibility of both the plant and the controller. A term in $R_{M S}$ is proper if the highest s-power in the denominator (polynomial or quasipolynomial), $s^{n}$, is higher or the same as the highest s-power in numerator, $s$, e.g. $n \geq l$.

\section{PARAMETERIZATION OF STABILIZING CONTROLLERS}

The above-introduced transfer function description of delayed systems over $R_{M S}$ ring is suitable in particular for algebraic control synthesis. Thus, let a singleinput single-output plant be estimated by an anisochronic model in the form

$$
\tilde{G}(s)=\frac{B(s)}{A(s)}=\frac{\frac{b(s)}{m(s)}}{\frac{a(s)}{m(s)}}
$$

where (quasi)polynomials $b(s)$ and $a(s)$ represent input-output plant behavior and dynamics and a selected stable (quasi)polynomial $m(s)$ makes $A(s)$ and $B(s)$ to be in $R_{M S}$, moreover, $A(s)$ and $B(s)$ are coprime - details about divisibility in $R_{M S}$ can be found in (Zítek and Kučera, 2003). Thus, consider the control loop as a simple feedback system depicted in Fig. 1.

Let a transfer function of the controller be $G_{R}(s)=$ $Q(s) / P(s)$. The aim of the control synthesis is to stabilize a feedback control system, obtain asymptotic tracking and attenuate load disturbance $d(t)$. Firstly, the stabilization of the feedback loop is guaranteed by solution of the Diophantine equation

$$
A(s) P_{0}(s)+B(s) Q_{0}(s)=1
$$




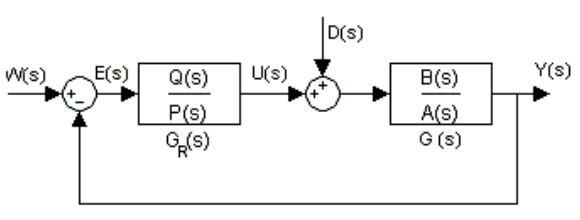

Fig. 1. Control feedback structure

where $P_{0}(s)$ and $Q_{0}(s)$ is a particular solution. Then, all stabilizing controllers can be expressed in a parametric form

$$
\frac{Q(s)}{P(s)}=\frac{Q_{0}(s)+A(s) Z(s)}{P_{0}(s)-B(s) Z(s)}, \quad P_{0}(s)-B(s) Z(s) \neq 0
$$

where $Z(s)$ is an arbitrary element of $R_{M S}$. The special choice of this element is capable of ensuring additional control conditions. Details and proofs for general ring can be found e.g. in (Vidyasagar, 1985; Kučera, 1993).

Secondly, conditions for asymptotic tracking and disturbance attenuation result from expression for $E(s)$ which reads

$$
E(s)=\frac{A(s) P(s)}{A(s) P(s)+B(s) Q(s)} W(s)-\frac{B(s) P(s)}{A(s) P(s)+B(s) Q(s)} D(s)
$$

where Laplace forms of reference and disturbance signals are $W(s)=H_{\mathrm{W}}(s) / F_{\mathrm{W}}(s)$ and $D(s)=$ $H_{\mathrm{D}}(s) / F_{\mathrm{D}}(s)$, and all numerators and denominators in both $W(s)$ and $D(s)$ are over $R_{M S}$. It is required that $E(s)$ must belong to $R_{M S}$, thus it is demanded that all unstable factors in both $F_{\mathrm{w}}(s)$ and $F_{\mathrm{D}}(s)$ divide $P(s)$. In practice, the most frequent case is both $w(t)$ and $d(t)$ are step functions, then the following condition for the absolute term in numerator of $P(s)$ is demanded

$$
\lambda\left(1-e^{-\tau s}\right)
$$

which indeed contains at least one zero pole. A free real parameter $\lambda \in \mathfrak{R}$ can be chosen as a multiple pole in the form $\lambda=m_{0}^{n}$ where $n$ is the order of the plant. Condition (8) is ensured by the suitable choice of $Z(s)$ in (6). ). If $w(t)$ or $d(t)$ are another functions, divisibility conditions can be more complex.

\section{CONTROLLER TUNING}

The whole system behavior is influenced by the choice of (quasi)polynomial $m(s)$. In order to simplify $m(s)$, it is usually chosen as the polynomial with multiple real stable pole $m_{0}$, i.e. $m(s)=\left(s+m_{0}\right)^{n}$. However, it follows from the above-mentioned facts that for unstable plants it is necessary to use a quasipolynomial instead of polynomials. Just and comprehensive solution to this problem has not been arrived at yet. In (Zítek et al., 2005), "a pole placement principle" is interpreted as a shifting of dominant poles. In this paper, "an equalization method" is adopted (e.g. in Klán, 2005). This method was originally derived for input-output delayed systems, nevertheless, in some limit approximation it seems to be useful also for anisochronic systems. According to this tuning approach, for the PI controller together with a first order plan it is postulated

$$
K_{P}=\frac{1}{K} \frac{1+(1-\sigma)^{2}}{2} \quad T_{I}=(T+\tau) \frac{1+(1-\sigma)^{2}}{2}
$$

where $K_{P}$ and $T_{I}$ are a gain and an integral time constant, respectively, $K$ and $T$ are a gain and a time constant of the plant, $\tau$ is time delay and $\sigma=\tau /(T+\tau)$. It is impossible to fulfil simultaneously both the conditions in (9), however satisfaction of $K_{P}$ gives better simulation results.

\section{ILLUSTRATIVE EXAMPLES}

\subsection{First order stable plants}

Let the plant be expressed by the anisochronic delayed first order model

$$
G_{S 1}(s)=\frac{K e^{-\tau s}}{T s+e^{-\theta s}}=\frac{\frac{K e^{-\tau s}}{s+m_{0}}}{\frac{T s+e^{-\theta s}}{s+m_{0}}}=\frac{B(s)}{A(s)}
$$

Parameters $K, T, \tau$ and $\theta$ can be acquired by relay experiment or via successive integration (Zítek and Vyhlídal, 2004). Using (5) the following, particular solution is obtained

$$
P_{0}(s)=\frac{s+m_{0}-K e^{-\tau s}}{T s+e^{-\theta s}} ; Q_{0}=1
$$

and if $Z(s)$ is chosen as in (12), then a controller satisfying both a reference tracking and a disturbance rejection has the transfer function (13).

$$
\begin{aligned}
& Z_{1}(s)=\frac{\kappa\left(s+m_{0}\right)}{T s+e^{-\theta s}} ; \kappa=\frac{m_{0}}{K}-1 \\
& G_{R 1}(s)=\frac{Q(s)}{P(s)}=\frac{m_{0}}{K} \frac{T s+e^{-\theta s}}{s+m_{0}\left(1-e^{-\tau s}\right)}
\end{aligned}
$$

The controller structure (13) can be easily compared with the well-known Smith predictor (Pekař and Prokop, 2006). For low frequencies, $s \rightarrow 0$, controller (13) works as a PI (proportionally-integrative) one. 
Assuming this steady-state behavior of the controller the equalization principle (9) may be taking into account. This idea results in a choice for $m_{0}$ as

$$
m_{0}=\frac{1+(1-\sigma)^{2}}{2 T}
$$

Hence, for example let a plant be modeled as $K=4.5$, $T=7, \tau=3, \theta=0.5$ and the ideal agreement of model and plant is presumed. The presented approach (5) (8) with respect to (14), $m_{0}=0.14$, results in the controller

$$
G_{R 1}(s)=0,031 \frac{7 s+e^{-0.5 s}}{s+0,14\left(1-e^{-3 s}\right)}
$$

To compare the influence of $m_{0}$ and the efficiency of the "equalization method", simulation results for various $\mathrm{m}_{0}$ values are depicted in Fig. 2 .

The following conditions were set for all simulations in this section: step reference value $w(t)=1$ for $\left.t \in<0 ; T_{S I M} / 3\right), w(t)=2$ for $\left.t \in<T_{S I M} / 3 ; T_{S I M}\right)$, step load disturbance $d(t)=-0.1$ for $t \geq 2 T_{S I M} / 3$, where $T_{S I M}$ is simulation time.
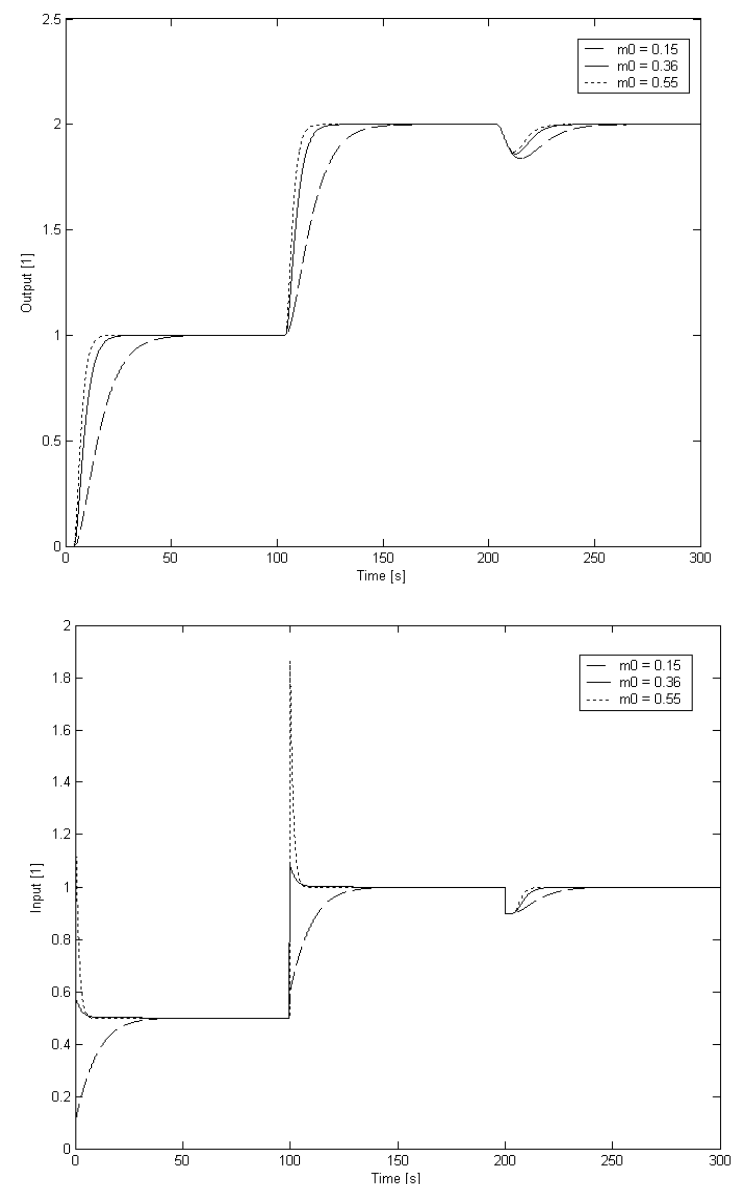

Fig. 2. Control of the first order stable plant (10) output and input for $m_{0}$ according to (14) and some other options
Obviously, as can be seen from the figures, the higher $m_{0}$ value implies faster and more "aggressive" behavior which can spoil an actuator and make the controller less robust.

\subsection{First order unstable plants}

Although many researches have implemented the $R_{M S}$ ring for the controller design, only stable plants were accentuated. Hereinafter, the controller design approach above presented is utilized also for an unstable first order plant. Hence, let the plant be modeled as

$$
G_{S 2}(s)=\frac{K e^{-\tau s}}{T s-e^{-\vartheta s}}=\frac{\frac{K e^{-\tau s}}{r_{0} K e^{-\tau s}+T s-e^{-\theta s}}}{\frac{T s-e^{-\theta s}}{r_{0} K e^{-\tau s}+T s-e^{-\theta s}}}
$$

where a scalar real parameter $r_{0}$ stabilize a common quasipolynomial denominator in (16), see details in (Pekař and Prokop, 2007). The stability can be checked by the Mikhailov criterion (Zítek, 1997). The solution of the Diophantine equation (6) is clearly $Q_{0}=r_{0}, P_{0}=1$. Selecting the parameterization factor

$$
Z_{2}(s)=\frac{m_{0}\left[r_{0} K e^{-\tau s}+T s-e^{-\theta s}\right]}{K\left(s+m_{0}\right)}
$$

leads to the primary controller $G_{R 2}(s)$ of the structure

$$
G_{R 2}(s)=\frac{\left(r_{0} K+m_{0} T\right) s+m_{0}\left[r_{0} K-e^{-\theta s}\right]}{K\left[s+m_{0}\left(1-e^{-\tau s}\right)\right]}
$$

Assuming steady-state approximation of (18) again, the PI structure is obtained and "equalization method" can be used. However, any attempt to fulfill the condition for $K_{P}$ according to (9) leads to negative $m_{0}$, which is not allowed due to the stability requirements. Thus, the condition for $T_{I}$ instead of $K_{P}$ must be taken in account, which results in

$$
\begin{aligned}
& m_{0}=\frac{r_{0} K(\delta-1)-\delta}{T} \\
& \delta=\frac{(T+\tau)^{2}+T^{2}}{2(T+\tau)}
\end{aligned}
$$

Consider the following plant parameters: $K=3, T=$ 5, $\tau=4, \theta=0.8$. Parameter $r_{0}$ can be taken as $r_{0}=$ 0.434 , i.e. stability gain margin is $K_{G}=1.3$. Formula (18) with respect to (19), $m_{0}=0.095$, leads to

$$
G_{R 2}(s)=\frac{1.775 s+0.124-0.095 e^{-0.8 s}}{3\left[s+0.095\left(1-e^{-4 s}\right)\right]}(20)
$$

Again, the suitability of the "equalization method" and the influence of $m_{0}$ are illustrated in Fig. 3. 

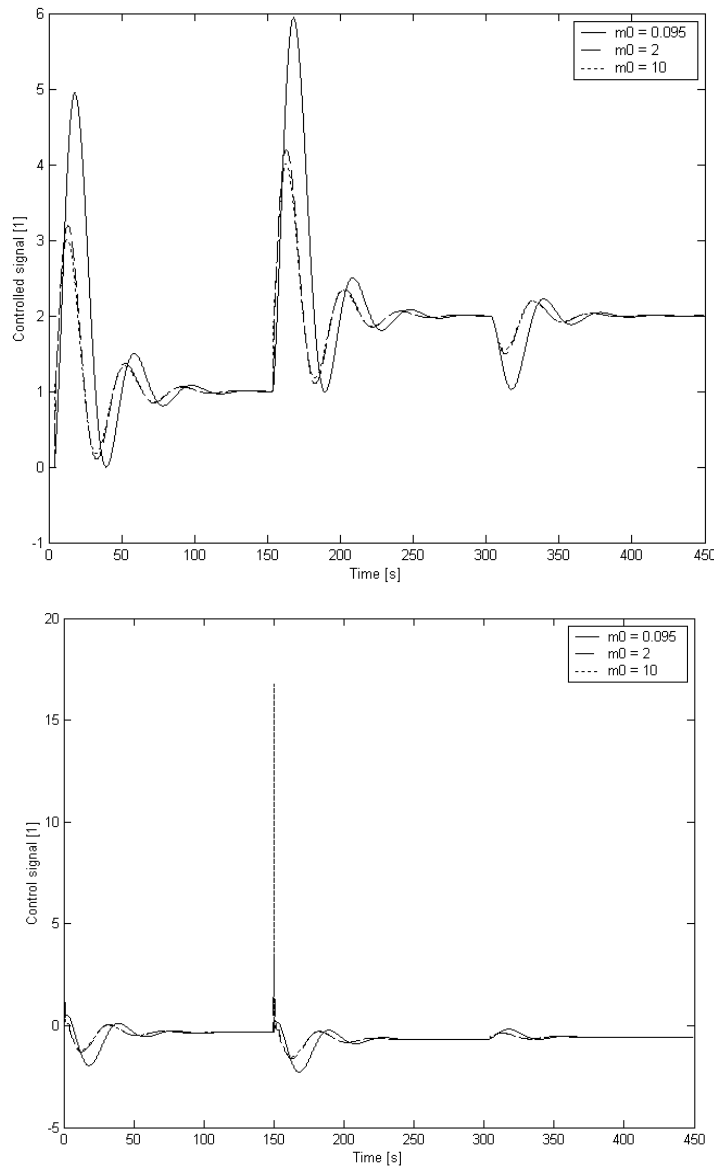

Fig. 3. Control of the first order unstable plant (16) output and input for $m_{0}$ according to (19) and some other options

Responses in these simulations are relatively slow in contrast to "unstable" time constant $T$ of the plant. The approach suffers from high overshoots after step reference signal changes. This overshoots can be attenuated by increasing $m_{0}$, which is sacrificed by faster changes of controlled signal.

The example opens up possibility of using the presented algebraic approach for unstable (anisochronic) delayed systems; nevertheless, it also demonstrates infelicity of the "equalization method" which is herein utilized after some frequency adjustment of a derived controller.

\subsection{Higher order approximation}

The transcendental character of anisochronic functions is able to estimate and approximate models of high order dynamics. The following example demonstrates this ability. Assume a plant exactly governed by the transfer function

$$
G_{S 3}(s)=\frac{e^{-3 s}}{(3 s+1)^{5}}
$$
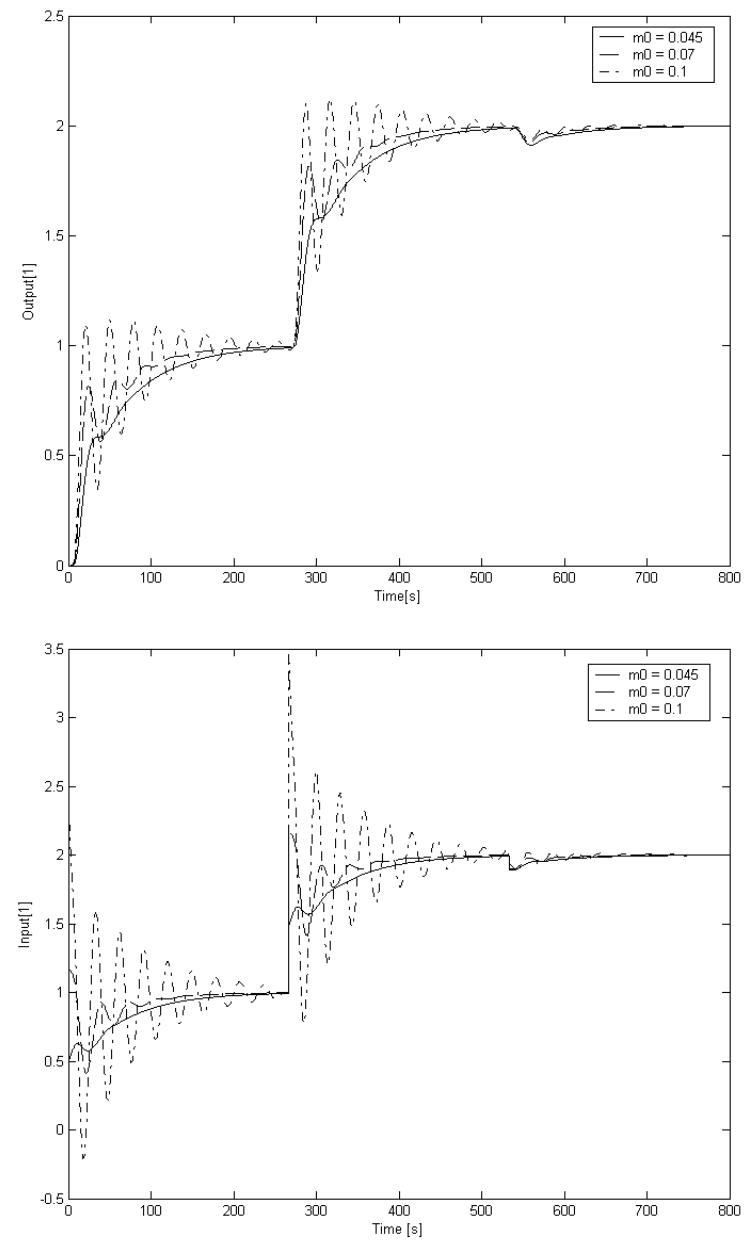

Fig. 4. Fifth order plant modeled by the first order anisochronic model (10) and controlled by (23) output and input for various $m_{0}$ values

After some autotuning experiments with asymmetric relay technique (see Prokop et al, 2006), this process can be approximated by the transfer function of form (10) as follows

$$
\hat{G}_{S 3}(s)=\frac{e^{-8.96 s}}{15.59 s+e^{-7.03 s}}
$$

The mentioned design approach according to (11) (13) results in the controller

$$
G_{R 3}(s)=\frac{Q(s)}{P(s)}=0.045 \frac{15.59 s+e^{-7.03 s}}{s+0.045\left(1-e^{-8.96 s}\right)}
$$

where the "tuning knob" $m_{0}$ is calculated according to (14) as $m_{0}=0.045$.

Figure 4 demonstrates efficiency of the controller in control plant (21). Simulation control responses of output and input system signals are displayed in the pictures, respectively. Various values of $m_{0}$ are chosen to verify the "equalization principle". 
The responses in Figure 4 clearly indicate that higher values of $m_{0}$ contribute to the faster but more oscillating control responses. Overall, the simulation responses are too slow in contrast to the step response of (21), where the settling time is approximately equal to $40 \mathrm{~s}$. The value $m_{0}=0.045$ calculated from (14) based on considerably modified "equalization method" gives quite satisfactory control response in comparison with other options of $m_{0}$. However, it is reasonable suspicious that frequency simplification of the controller can notably contribute to uselessness of the mentioned tuning method.

Model (10) is capable of describing in particular nonoscillatory plants. This feature is due to absence of more descriptive parameters in the model, especially in the nominator. Thus, for systems of more complex dynamics it is better to choose a different anisochronic model which has more parameters. However, this choice involves an investigation of suitable identification techniques.

\section{CONCLUSIONS}

The contribution is focused on algebraic control approach in the special ring of proper and stable RQ meromorphic functions $\left(R_{M S}\right)$. A delayed plant is described as a ratio of two terms in $R_{M S}$. Both the input-output delayed systems and anisochronic (dynamic-delayed) systems are assumed. For the plant model, the control synthesis is then performed through a solution of a Diophantine equation in this ring. The methodology generates a class of Smithlike delay compensating controllers. The design method brings a scalar tuning parameter $m_{0}>0$ that can be adjusted by various strategies; the "equalization method" can be one of them. The methodology is illustrated by the simulation examples for stable and unstable first order systems. Higher order dynamics approximation using anisochronic model is also presented in the example. The simulations show simplicity and usability of the proposed methodology for delayed systems. For further study, the problem of anisochronic model estimation and validation and searching of suitable controller tuning method is opened.

\section{ACKNOWLEDGEMENT}

This work was supported in part by the grants of Ministry of Education, Youth and Sports of the Czech Republic MSM 7088352102.

\section{REFERENCES}

Kamen, E.W. (1978). An operator theory of linear functional differential equations. Journal of Differential Equations, 27, 274-297.

Klán, P. (2005). PI controllers with good tuning (in Czech). Automatizace, 10, 53 - 55.
Kučera, V. (1993). Diophantine equations in control A survey. Automatica, 29, 1361-75.

Pekař, L. (2005). Simulation and control of delayed systems (in Czech). Graduation theses, 88 p., Faculty of Technology, Tomas Bata University in Zlín.

Pekař, L. and R. Prokop (2006). Use of meromorphic functions in the control design for delayed systems. In: Proceedings of the 7th International Scientific - Technical Conference Process Control 2006, p. 50. Kouty nad Desnou, Czech Republic.

Pekař, L. and R. Prokop (2007). A simple stabilization and algebraic control design of unstable delayed systems using meromorphic functions. In: Proceedings of the $26^{\text {th }}$ IASTED International Conference MIC 2007, pp. 183188. Innsbruck, Austria.

Prokop, R., L. Pekař and J. Korbel (2006). Relaybased autotuning with meromorphic control synthesis. In: Proceedings of the 6th Asian Control Conference, pp. 376-382. Bali, Indonesia.

Vidyasagar, M. (1985). Control system synthesis: a factorization approach. MIT Press, Cambridge, M.A.

Vyhlídal, T. and P. Zítek (2001). Control system design based on a universal first order model with time delays. In: Proceedings of $2^{\text {nd }}$ International Conference on Advanced Engineering Design. Glasgow: University of Glasgow, and Acta Polytechnica, 41, 49-53.

Zítek, P. (1997). Frequency domain synthesis of hereditary control systems via anisochronic state space. International Journal of Control, 66.

Zítek, P. and V. Kučera (2003). Algebraic design of anisochronic controllers for time delay systems. International Journal of Control, 76, 905-921.

Zítek, P. and A. Víteček (1999). The design of control of subsystems with delays and nonlinearities (in Czech). ČVUT publishing, Prague.

Zítek, P. and T. Vyhlídal (2002). Dominant eigenvalue placement for time delay systems. In: Proceedings of CONTROLO 2002, $5^{\text {th }}$ Portuguese Conference on Automatic Control. University of Aveiro, Portugal.

Zítek, P. and Vyhlídal, T. (2003). Low order time delay approximation of conventional linear model. In: Proceedings of $4^{\text {th }}$ MathMod conference. Vienna, Austria.

Zítek, P., V. Kučera, and T. Vyhlídal (2005). Meromorphic Stabilization and Control of Time Delay Systems. In: Preprints of the $16^{\text {th }}$ World Congress of the International Federation of Automatic Control [CD-ROM]. Prague, IFAC. 